\title{
A Wave-Based Vibration Analysis of a Finite Timoshenko Locally Resonant Beam Suspended with Periodic Uncoupled Force-Moment Type Resonators
}

\author{
Hangyuan Lv ${ }^{1, *(\mathbb{C}) \text { and Yimin Zhang }}{ }^{2}$ \\ 1 School of Mechanical Engineering and Automation, Northeastern University, Shenyang 110819, China \\ 2 Equipment Reliability Institute, Shenyang University of Chemical Technology, Shenyang 110142, China; \\ zhangyimin@syuct.edu.cn \\ * Correspondence: lvhy@me.neu.edu.cn; Tel.: +86-24-8367-3816
}

Received: 9 November 2020; Accepted: 9 December 2020; Published: 11 December 2020

\begin{abstract}
This paper first employs and develops an exact wave-based vibration analysis approach to investigate a finite Timoshenko beam carrying periodic two-degree-of-freedom (2-DOF) uncoupled force-moment type resonators. In the approach, vibrations are described as structural waves that propagate along uniform structural elements and reflected and transmitted at structural discontinuities. Each uncoupled force-moment type resonator is considered as a cell which injects waves into the distributed beam through the transverse force and the bending moment at the attached point. By assembling wave relations of the cells into the beam, the forced vibration problem of the locally resonant (LR) structure is turned to be the solution to a related set of matrix equations. In addition, the parametric analysis provides an efficient method to obtain wide low-frequency range band-gaps. Accuracy of the proposed wave-based vibration analysis approach is demonstrated by the simulated and measured results of two sets of beam-like resonator samples.
\end{abstract}

Keywords: locally resonant phononic crystal; uncoupled force-moment resonator; Timoshenko beam; wave-based vibration analysis

\section{Introduction}

In the past decade, the propagation of elastic or acoustic waves in periodic structures, known as phononic crystals (PCs) and phononic metamaterials (PMs), has received growing attention. Such structures are attractive to be used in numerous engineering applications, such as vibration isolators, frequency sensors, sound barriers, acoustic cloaks, vibration energy harvesters, etc. [1-4], because of the existence of elastic band-gap properties within which no flexural wave can propagate through. Particularly, wide and low-frequency band-gaps are mostly desired in noise and vibration control engineering.

Nowadays, plenty of studies are focused on locally resonant (LR) PCs due to their advantage in achieving low-frequency band-gaps with small lattice constants. It is worth mentioning that a beam carrying one or several spring-mass systems, such as oscillators or vibration absorbers, known as an LR beam, is frequently encountered in the fields of mechanical, civil and aeronautical engineering [5].

Many methods have been employed to study the band-gap properties of LR beams. Yu presented an Euler-Bernoulli beam [6] and a Timoshenko beam [7] suspended with a periodic resonator base on a transfer matrix (TM) method. The TM method is the most widely applied in analysis of the infinite LR beams [6-10]. Xiao [11] studied an LR beam using an analytical method based on the spectral element method. Hajhosseini [12] studied an infinite Euler-Bernoulli LR beam consisting of concentrated rigid masses and tapered beam elements with a linearly variable width using a generalized differential 
quadrature method. Liang [13] proposed an improved differential quadrature method to obtain the band-gap properties of a Euler-Bernoulli LR beam with spring-mass resonators. Lepidi [14] studied a kind of nonlinear LR beam under the micro-scale using the method of multiple scales. However, band-gap properties of finite structures are more important in practical engineering applications. So far, a finite element method (FEM) is the only method which is widely used in analysis of the finite LR beams $[6,7,9,11,12,15]$. Although FEM is suitable for plenty of structures, it is restricted to trained personnel and is commercially expensive, in particular for preliminary design evaluation since the model has to be rebuilt if the parameter is changed. Fast and low-cost analysis approach is more desired for finite structures.

More recently, Mei [16] first proposed a finite Euler-Bernoulli beam carrying a single two-degree-of-freedom (2-DOF) spring-mass resonator using a wave-based vibration analysis approach. It is a very useful vibration analysis approach that provides the exact solution for structural waves that propagate along uniform structural elements and are reflected and transmitted at structural discontinuities, such as joints, resonators, loading sites, and boundaries [17-21]. Furthermore, Leamy [22] employed the exact wave-based analysis approach to investigate wave propagation in periodic structures. The approach is used for determining natural frequencies, mode shapes, and forced response in structures composed of slender members through an exact procedure. In the approach, the relationships and undetermined coefficients of wave propagation between each uniform beam section are obtained through analysis of the reflection and transmission of waves at discontinuities. With the relations, in combing with MATLAB software R2018b (MathWorks Inc., Natick, MA, USA), a general-purposed code can be written for assembling all the necessary discontinuity conditions into a single set of matrix equations. With the general code, the model can be built and modified automatically and easily.

This paper first employs and develops the wave-based vibration analysis approach to study a finite Timoshenko LR beam suspended with periodic uncoupled force-moment type resonators. The resonator is 2-DOF. As described in Ref. [23], it exerts an uncoupled transverse force and a bending moment to the host beam, attenuating both the translational and rotational motions-it therefore has broader band-gap than a 1-DOF one. The goal of this paper is to provide an efficient and exact method to analyze the finite LR structures. The frequency response functions and band-gap properties are acquired through forced vibration analysis. Moreover, the parametric study is performed to illustrate the effect of mass and length of moment arm of the resonator on the width of the low-frequency band-gap. Finally, the accuracy of the approach is validated through comparisons to simulation and experimental results.

\section{Wave-Based Vibration Analysis Approach}

\subsection{Overview}

For Timoshenko beams, the solutions from the equations of motion governing bending, rotational, and longitudinal wave propagation are given by [24],

$$
\begin{gathered}
G A \kappa\left(\frac{\partial \psi(x, t)}{\partial x}-\frac{\partial^{2} w(x, t)}{\partial x^{2}}\right)+\rho A \frac{\partial^{2} w(x, t)}{\partial t^{2}}=q(x, t) \\
E I \frac{\partial^{2} \psi(x, t)}{\partial x^{2}}+G A \kappa\left(\frac{\partial w(x, t)}{\partial x}-\psi(x, t)\right)-\rho I \frac{\partial^{2} \psi(x, t)}{\partial t^{2}}=0 \\
\rho A \frac{\partial^{2} u(x, t)}{\partial t^{2}}-E A \frac{\partial^{2} u(x, t)}{\partial x^{2}}=p(x, t)
\end{gathered}
$$

where $x$ denotes position along the neutral axis of beam, $t$ represents time, $w(x, t)$ and $u(x, t)$ are the transverse and axial deflections, respectively. $\psi(x, t)$ denotes the total cross-sectional rotational angle, $\frac{\partial w(x, t)}{\partial x}$ is the slope of the centerline, and $\frac{\partial w(x, t)}{\partial x}-\psi(x, t)$ is the shear angle. Loads $q(x, t)$ and 
$p(x, t)$ denote the external transverse and longitudinal forces applied per unit length, respectively. The material properties are mass density $\rho$, Young's modulus $E$, and shear modulus $G$. The geometrical properties are cross-sectional area $A$, area moment of inertia $I$, and shear coefficient $\kappa$. The expressions of shear force $V(x, t)$, bending moment $M(x, t)$, and longitudinal force $F(x, t)$, respectively, are,

$$
\begin{gathered}
V(x, t)=G A \kappa\left(\frac{\partial w(x, t)}{\partial x}-\psi(x, t)\right) \\
M(x, t)=E I \frac{\partial \psi(x, t)}{\partial x} \\
F(x, t)=E A \frac{\partial u(x, t)}{\partial x}
\end{gathered}
$$

In the absence of loading and with the time dependence $e^{i \omega t}$ suppressed, the solutions to the free wave propagation Equations (1)-(3) can be expressed as,

$$
\begin{gathered}
w(x, t)=a_{1}^{+} \mathrm{e}^{-i k_{1} x}+a_{2}^{+} \mathrm{e}^{k_{2} x}+a_{1}^{-} \mathrm{e}^{i k_{1} x}+a_{2}^{-} \mathrm{e}^{k_{2} x} \\
\psi(x, t)=-i P a_{1}^{+} e^{-i k_{1} x}-N a_{2}^{+} e^{-k_{2} x}+i P a_{1}^{-} e^{i k_{1} x}+N a_{2}^{-} e^{k_{2} x} \\
u(x, t)=c^{+} \mathrm{e}^{-i k_{3} x}+c^{-} \mathrm{e}^{i k_{3} x}
\end{gathered}
$$

in which, $a_{1}, a_{2}$ and $c$ denote the amplitude of the propagating flexural wave, the near-field flexural wave, and the propagating longitudinal wave, respectively. The superscripts + or - denote the forwardor backward-propagating waves. $k_{1}, k_{2}, k_{3}$ denote the three wavenumbers. $i P$ and $N$ in the equations relate the rotational solution to the transverse displacement solution and are given by,

$$
P=k_{1}\left(1-\frac{\omega^{2}}{k_{1}^{2} C_{s}^{2}}\right), \quad N=k_{2}\left(1+\frac{\omega^{2}}{k_{2}^{2} C_{s}^{2}}\right)
$$

The wavenumber-frequency dispersion relationships are,

$$
\begin{gathered}
k_{1}=\sqrt{\frac{1}{2}\left[\left(\frac{1}{C_{s}}\right)^{2}+\left(\frac{C_{r}}{C_{b}}\right)^{2}\right] \omega^{2}+\sqrt{\left(\frac{\omega}{C_{b}}\right)^{2}+\frac{1}{4}\left[\left(\frac{1}{C_{s}}\right)^{2}-\left(\frac{C_{r}}{C_{b}}\right)^{2}\right]^{2} \omega^{4}}} \\
k_{2}=\sqrt{-\frac{1}{2}\left[\left(\frac{1}{C_{s}}\right)^{2}+\left(\frac{C_{r}}{C_{b}}\right)^{2}\right] \omega^{2}+\sqrt{\left(\frac{\omega}{C_{b}}\right)^{2}+\frac{1}{4}\left[\left(\frac{1}{C_{s}}\right)^{2}-\left(\frac{C_{r}}{C_{b}}\right)^{2}\right]^{2} \omega^{4}}} \\
k_{3}=\sqrt{\frac{E}{\rho} \omega^{2}}
\end{gathered}
$$

where the wave speeds for bending, shear and rotation are given as,

$$
C_{s}=\sqrt{\frac{G A \kappa}{\rho A}}, C_{b}=\sqrt{\frac{E I}{\rho A}}, C_{r}=\sqrt{\frac{I}{A}}
$$

For the vibration analysis of the periodic uncoupled force-moment resonators attached on the beam in this paper, there are only bending vibrations involved.

\subsection{Propagation Matrix}

Between discontinuities, the solutions Equations (7)-(9) exactly represents the state of wave propagation at a single frequency in a uniform beam. As shown in Figure 1, considering two points A 
and $\mathrm{B}$ of a beam falling between discontinuities and separated by distance $x$. As there are only bending vibrations involved, the propagation matrix can be defined relating coefficients at $A$ to $B$ as,

$$
b^{+}=f(x) a^{+}, a^{-}=f(x) b^{-}
$$

where,

$$
\begin{aligned}
& \boldsymbol{a}^{+}=\left[\begin{array}{l}
a_{1}+ \\
a_{2}+
\end{array}\right], \boldsymbol{a}^{-}=\left[\begin{array}{l}
a_{1}^{-} \\
a_{2}^{-}
\end{array}\right], \boldsymbol{b}^{+}=\left[\begin{array}{l}
b_{1}^{+} \\
b_{2}+
\end{array}\right], \boldsymbol{b}^{-}=\left[\begin{array}{l}
b_{1}^{-} \\
b_{2}^{-}
\end{array}\right] \\
& \mathrm{a}^{+} \underset{\mathrm{A}}{\longrightarrow} \mathrm{a}^{-\longleftarrow} \mathrm{b}^{+} \\
& \longleftarrow \mathrm{b}^{-}
\end{aligned}
$$

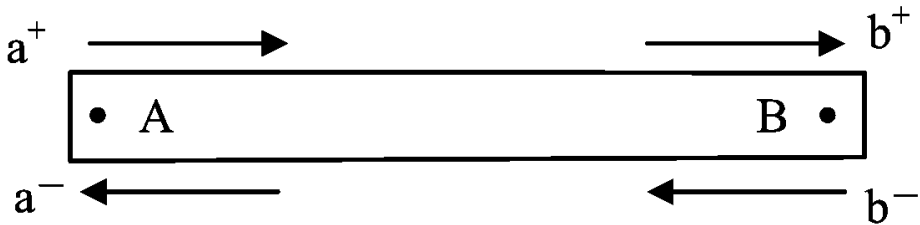

Figure 1. Wave propagation at two points separated by a distance $x$ along a uniform beam.

$a^{+}$and $a^{-}$denote the wave coefficients for forward and backward propagating waves at point A. $\boldsymbol{b}^{+}$and $\boldsymbol{b}^{-}$are wave coefficients at point $B$.

$$
f(x)=\left[\begin{array}{cc}
e^{-i k_{1} x} & 0 \\
0 & e^{-k_{2} x}
\end{array}\right]
$$

\subsection{Reflection at a Free Support}

For free boundary conditions, the transverse force and bending moment must all vanish. A reflection matrix can be derived with these conditions, which relates the incident waves $\boldsymbol{a}^{+}$ to the reflected waves $a^{-}$,

$$
a^{-}=r_{f} a^{+}
$$

where

$$
r_{f}=\left[\begin{array}{ll}
\frac{-P k_{1}\left(-N+k_{2}\right)+i k_{2} N\left(k_{1}-P\right)}{P k_{1}\left(-N+k_{2}\right)+i k_{2} N\left(k_{1}-P\right)} & \frac{2 N k_{2}\left(-N+k_{2}\right)}{P k_{1}\left(-N+k_{2}\right)+i k_{2} N\left(k_{1}-P\right)} \\
\frac{2 i P k_{1}\left(-P+k_{1}\right)}{P k_{1}\left(-N+k_{2}\right)+i k_{2} N\left(k_{1}-P\right)} & \frac{P k_{1}\left(-N+k_{2}\right)-i k_{2} N\left(k_{1}-P\right)}{P k_{1}\left(-N+k_{2}\right)+i k_{2} N\left(k_{1}-P\right)}
\end{array}\right]
$$

\subsection{Analysis of a Beam Carrying an Uncoupled Force-Moment Type Resonator}

Figure 2 shows a beam suspended with periodic uncoupled force-moment type resonators. In the figure, $m_{A}$ and $m_{B}$ present the masses of the resonators, $k_{A}$ and $k_{B}$ are the stiffnesses of two linear elastic springs, $a$ and $b$ denote the lengths of the moment arm of the resonators, and $L$ is the lattice constant (spacing between two adjacent resonators) of the periodic structure.

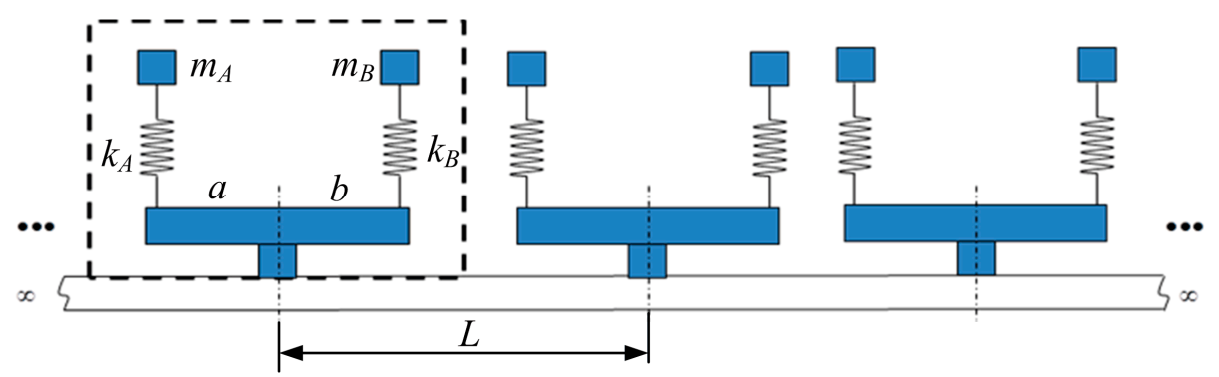

Figure 2. A beam with periodic uncoupled force-moment type resonators. 
Figure 3 depicts the free body diagram of a single resonator on the beam. Two springs are connected to the beam at points $\mathrm{A}$ and $\mathrm{B}$, respectively. The transverse deflections of the mass blocks $m_{A}$ and $m_{B}$ are denoted as $w_{A m}$ and $w_{B m}$. The transverse deflection, axial deflection and angular rotation of the host beam are $w, u$, and $\psi$. As only the bending vibrations involved in the system, the axial deflection $u$ is not considered below. The uncoupled force-moment resonator is located on the host beam at point $\mathrm{O}$ with a rigid block. Thus, the angular rotation of the arms $\mathrm{AB}$ is $\psi$ as well.

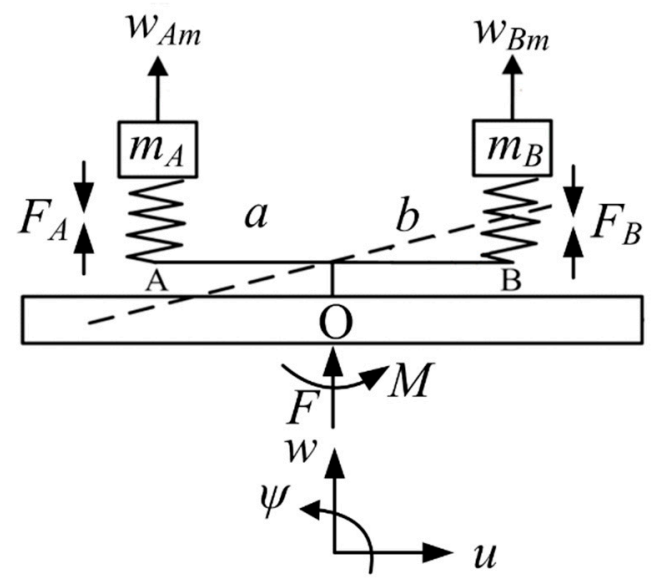

Figure 3. Free body diagram of a single uncoupled force-moment type resonator.

Assuming the directions of the deflections are as shown in Figure 3, the equation of motion of the resonator can be written as follows,

$$
\begin{aligned}
& -F_{A}=m_{A} \ddot{w}_{A m} \\
& -F_{B}=m_{B} \ddot{w}_{B m}
\end{aligned}
$$

where

$$
\begin{gathered}
F_{A}=k_{A}\left(w_{A m}-w+a \psi\right) \\
F_{B}=k_{B}\left(w_{B m}-w-b \psi\right)
\end{gathered}
$$

Considering time harmonic motion of the system with frequency $\omega$, and substituting Equations (22) and (23) into Equations (20) and (21), the displacements of the mass blocks $w_{A m}$ and $w_{B m}$ can be expressed in terms of the deflections of point $\mathrm{O}$ on the host beam $w$ and $\psi$ as,

$$
\begin{aligned}
& w_{A m}=\frac{k_{A}}{k_{A}-m_{A} \omega^{2}}(w-a \psi) \\
& w_{B m}=\frac{k_{B}}{k_{B}-m_{B} \omega^{2}}(b \psi+w)
\end{aligned}
$$

Substituting Equations (24) and (25) back into Equations (22) and (23), the forces $F_{A}$ and $F_{B}$ can be expressed with $w$ and $\psi$ as,

$$
\begin{aligned}
& F_{A}=\frac{k_{A} m_{A} \omega^{2}}{k_{A}-m_{A} \omega^{2}}(w-a \psi) \\
& F_{B}=\frac{k_{B} m_{B} \omega^{2}}{k_{B}-m_{B} \omega^{2}}(b \psi+w)
\end{aligned}
$$

At point $\mathrm{O}$ on the host beam, the transverse force $F$ and bending moment $M$ applied by the resonator are,

$$
\begin{gathered}
F=F_{A}+F_{B} \\
M=F_{B} b-F_{A} a
\end{gathered}
$$


Substituting Equations (26) and (27) into Equations (28) and (29), one obtains the transverse force $F$ and bending moment $M$ insert to the host beam from the uncoupled force-moment resonator as,

$$
\begin{gathered}
F=r_{11} w+r_{12} \psi \\
M=r_{21} w+r_{22} \psi
\end{gathered}
$$

where

$$
\begin{gathered}
r_{11}=\frac{k_{A} m_{A} \omega^{2}}{k_{A}-m_{A} \omega^{2}}+\frac{k_{B} m_{B} \omega^{2}}{k_{B}-m_{B} \omega^{2}} \\
r_{12}=r_{21}=\frac{k_{B} m_{B} \omega^{2}}{k_{B}-m_{B} \omega^{2}} b-\frac{k_{A} m_{A} \omega^{2}}{k_{A}-m_{A} \omega^{2}} a \\
r_{22}=\frac{k_{B} m_{B} \omega^{2}}{k_{B}-m_{B} \omega^{2}} b^{2}+\frac{k_{A} m_{A} \omega^{2}}{k_{A}-m_{A} \omega^{2}} a^{2}
\end{gathered}
$$

The relation between the transverse force and bending moment generated by the resonator and the deflections of the beam is described in Equations (30) and (31).

\subsection{Applied Forces and Moments}

The transverse force and bending moments generated by the resonator inject waves to the host beam. As shown in Figure 4, a point transverse force $F$, and a bending moment $M$, are assumed to be harmonic with frequency $\omega$, respectively, applied at $x=0$, and the generated waves $a$ and $b$. Continuity and equilibrium conditions result in expressions relating wave coefficients to the right of loading to those to the left,

$$
\begin{gathered}
b^{+}-a^{+}=f+m \\
b^{-}-a^{-}=-f+m
\end{gathered}
$$

where the vectors of the excited wave amplitudes are related to the applied loading by,

$$
\begin{aligned}
& \boldsymbol{q}=\left[\begin{array}{c}
i N \\
P
\end{array}\right] \frac{F}{\operatorname{GA\kappa }\left(k_{2} P-k_{1} N\right)} \\
& \boldsymbol{m}=\left[\begin{array}{c}
-1 \\
1
\end{array}\right] \frac{M}{2 E I\left(k_{1} P+k_{2} N\right)}
\end{aligned}
$$

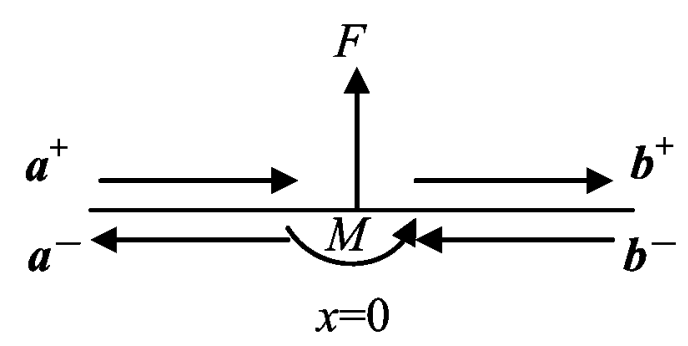

Figure 4. Waves generated by external forces and moment.

Combining Equations (7)-(9) and (30)-(38), the following equations describe the relations of vibration waves at discontinuities caused by the uncoupled force-moment resonator attachment,

$$
\left[\begin{array}{ll}
A_{11} & A_{12} \\
A_{21} & A_{22}
\end{array}\right]\left[\begin{array}{l}
a^{+} \\
a^{-}
\end{array}\right]+\left[\begin{array}{ll}
B_{11} & B_{12} \\
B_{21} & B_{22}
\end{array}\right]\left[\begin{array}{l}
b^{+} \\
b^{-}
\end{array}\right]=0
$$


The coefficient matrices of Equation (39) are as follows,

$$
\begin{gathered}
A_{\mathbf{1 1}}=\left[\begin{array}{cc}
i N \beta_{1}\left(r_{11}-i P r_{12}\right)-\beta_{2}\left(r_{21}-i P r_{22}\right)+1 & i N \beta_{1}\left(r_{11}-N r_{12}\right)-\beta_{2}\left(r_{21}-N r_{22}\right) \\
P \beta_{1}\left(r_{11}-i P r_{12}\right)+\beta_{2}\left(r_{21}-i P r_{22}\right) & P \beta_{1}\left(r_{11}-N r_{12}\right)+\beta_{2}\left(r_{21}-N r_{22}\right)+1
\end{array}\right] \\
A_{\mathbf{1 2}}=\left[\begin{array}{cc}
i N \beta_{1}\left(r_{11}+i P r_{12}\right)-\beta_{2}\left(r_{21}+i P r_{22}\right) & i N \beta_{1}\left(r_{11}+N r_{12}\right)-\beta_{2}\left(r_{21}+N r_{22}\right) \\
P \beta_{1}\left(r_{11}+i P r_{12}\right)+\beta_{2}\left(r_{21}+i P r_{22}\right) & P \beta_{1}\left(r_{11}+N r_{12}\right)+\beta_{2}\left(r_{21}+N r_{22}\right)
\end{array}\right] \\
A_{\mathbf{2 1}}=\left[\begin{array}{cc}
-i N \beta_{1}\left(r_{11}-i P r_{12}\right)-\beta_{2}\left(r_{21}-i P r_{22}\right) & -i N \beta_{1}\left(r_{11}-N r_{12}\right)-\beta_{2}\left(r_{21}-N r_{22}\right) \\
-P \beta_{1}\left(r_{11}-i P r_{12}\right)+\beta_{2}\left(r_{21}-i P r_{22}\right) & -P \beta_{1}\left(r_{11}-N r_{12}\right)+\beta_{2}\left(r_{21}-N r_{22}\right)
\end{array}\right] \\
A_{\mathbf{2 2}}=\left[\begin{array}{cc}
-i N \beta_{1}\left(r_{11}+i P r_{12}\right)-\beta_{2}\left(r_{21}+i P r_{22}\right)+1 & -i N \beta_{1}\left(r_{11}+N r_{12}\right)-\beta_{2}\left(r_{21}+N r_{22}\right) \\
-P \beta_{1}\left(r_{11}+i P r_{12}\right)+\beta_{2}\left(r_{21}+i P r_{22}\right) & -P \beta_{1}\left(r_{11}+N r_{12}\right)+\beta_{2}\left(r_{21}+N r_{22}\right)+1
\end{array}\right] \\
\boldsymbol{B}_{\mathbf{1 1}}=\boldsymbol{B}_{\mathbf{2 2}}=\left[\begin{array}{cc}
-1 & 0 \\
0 & -1
\end{array}\right] \\
\boldsymbol{B}_{\mathbf{1 2}}=\boldsymbol{B}_{21}=\left[\begin{array}{cc}
0 & 0 \\
0 & 0
\end{array}\right] \\
\beta_{1}=\frac{1}{2(G A \kappa)\left(k_{2} P-k_{1} N\right)} \\
\beta_{2}=\frac{1}{2(E I)\left(k_{1} P+k_{2} N\right)}
\end{gathered}
$$

\section{Forced Vibration Analysis of a LR Beam Using the Wave-Based Approach}

Based on the approach above, a finite Timoshenko beam suspended with eight periodic uncoupled force-moment type resonators was chosen for the assessing forced response and band-gap properties of the LR system. Figure 5 depicts vibration waves in the continuous beam. The structure is composed eight periodic uncoupled force-moment type resonators attached at point B, C, D, E, F, H, J, and K with lattice constant $L$. Two free supports are at point $A$ and $N$. The loading is applied at point $G$ with a distance $L_{11}$ from point $\mathrm{A}$ (left end of the host beam), and $L_{12}$ from point $\mathrm{B}$ (first resonator attached point). The distance from the last resonator to the right end of the host beam is $L_{2}$. The transmission and reflection relations of the waves in the beam elements are as follows.

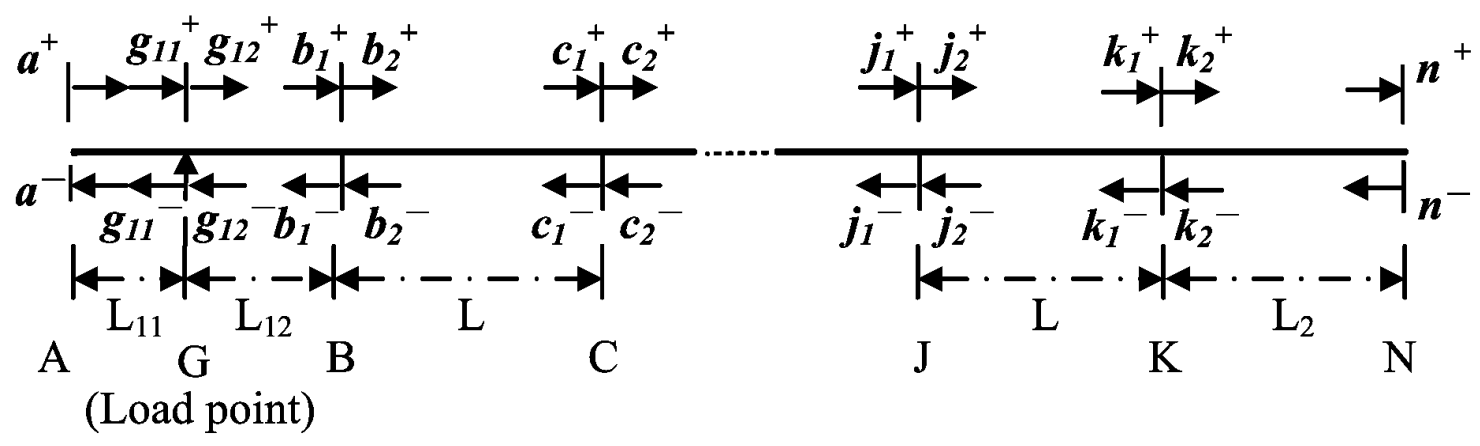

Figure 5. Wave analysis of a finite locally resonant (LR) beam.

At eight resonator attached points B, C, D, E, F, H, J, and K

$$
\begin{aligned}
& {\left[\begin{array}{ll}
A_{11} & A_{12} \\
A_{21} & A_{22}
\end{array}\right]\left[\begin{array}{l}
b_{1}^{+} \\
b_{1}
\end{array}\right]+\left[\begin{array}{ll}
B_{11} & B_{12} \\
B_{21} & B_{22}
\end{array}\right]\left[\begin{array}{l}
b_{2}^{+} \\
b_{2}^{-}
\end{array}\right]=0} \\
& {\left[\begin{array}{ll}
A_{11} & A_{12} \\
A_{21} & A_{22}
\end{array}\right]\left[\begin{array}{l}
c_{1}^{+} \\
c_{1}^{-}
\end{array}\right]+\left[\begin{array}{ll}
B_{11} & B_{12} \\
B_{21} & B_{22}
\end{array}\right]\left[\begin{array}{l}
c_{2}^{+} \\
c_{2}^{-}
\end{array}\right]=0} \\
& {\left[\begin{array}{ll}
A_{11} & A_{12} \\
A_{21} & A_{22}
\end{array}\right]\left[\begin{array}{l}
d_{1}^{+} \\
d_{1}^{-}
\end{array}\right]+\left[\begin{array}{ll}
B_{11} & B_{12} \\
B_{21} & B_{22}
\end{array}\right]\left[\begin{array}{l}
d_{2}^{+} \\
d_{2}^{-}
\end{array}\right]=0}
\end{aligned}
$$




$$
\begin{aligned}
& {\left[\begin{array}{ll}
A_{11} & A_{12} \\
A_{21} & A_{22}
\end{array}\right]\left[\begin{array}{l}
e_{1}^{+} \\
e_{1}^{-}
\end{array}\right]+\left[\begin{array}{ll}
B_{11} & B_{12} \\
B_{21} & B_{22}
\end{array}\right]\left[\begin{array}{l}
e_{2}^{+} \\
e_{2}^{-}
\end{array}\right]=0} \\
& {\left[\begin{array}{ll}
A_{11} & A_{12} \\
A_{21} & A_{22}
\end{array}\right]\left[\begin{array}{l}
f_{1}^{+} \\
f_{1}^{-}
\end{array}\right]+\left[\begin{array}{ll}
B_{11} & B_{12} \\
B_{21} & B_{22}
\end{array}\right]\left[\begin{array}{l}
f_{2}^{+} \\
f_{2}^{-}
\end{array}\right]=0} \\
& {\left[\begin{array}{ll}
A_{11} & A_{12} \\
A_{21} & A_{22}
\end{array}\right]\left[\begin{array}{l}
h_{1}^{+} \\
h_{1}^{-}
\end{array}\right]+\left[\begin{array}{ll}
B_{11} & B_{12} \\
B_{21} & B_{22}
\end{array}\right]\left[\begin{array}{l}
h_{2}^{+} \\
h_{2}^{-}
\end{array}\right]=0} \\
& {\left[\begin{array}{ll}
A_{11} & A_{12} \\
A_{21} & A_{22}
\end{array}\right]\left[\begin{array}{l}
j_{1}^{+} \\
j_{1}^{-}
\end{array}\right]+\left[\begin{array}{ll}
B_{11} & B_{12} \\
B_{21} & B_{22}
\end{array}\right]\left[\begin{array}{l}
j_{2}^{+} \\
j_{2}^{-}
\end{array}\right]=0} \\
& {\left[\begin{array}{ll}
A_{11} & A_{12} \\
A_{21} & A_{22}
\end{array}\right]\left[\begin{array}{l}
k_{1}^{+} \\
k_{1}^{-}
\end{array}\right]+\left[\begin{array}{ll}
B_{11} & B_{12} \\
B_{21} & B_{22}
\end{array}\right]\left[\begin{array}{l}
k_{2}^{+} \\
k_{2}^{-}
\end{array}\right]=0}
\end{aligned}
$$

At free end boundaries $\mathrm{A}$ and $\mathrm{N}$

$$
\begin{aligned}
& a^{+}=r_{f} a^{-} \\
& n^{-}=r_{f} n^{+}
\end{aligned}
$$

The forced vibration analysis of the LR beam has ten pairs of propagation relations along the beam elements AG, GB, BC, CD, DE, EF, FH, HJ, JK and KN.

Along BC

$$
c_{1}{ }^{+}=f(L) b_{2}{ }^{+}, b_{2}^{-}=f(L) c_{1}^{-}
$$

Along CD

$$
d_{1}^{+}=f(L) c_{2}{ }^{+}, c_{2}^{-}=f(L) d_{1}^{-}
$$

Along DE

$$
e_{1}^{+}=f(L) d_{2}^{+}, d_{2}^{-}=f(L) e_{1}^{-}
$$

Along EF

$$
f_{1}^{+}=f(L) e_{2}{ }^{+}, e_{2}{ }^{-}=f(L) f_{1}^{-}
$$

Along FH

$$
h_{1}^{+}=f(L) f_{2}^{+}, f_{2}^{-}=f(L) h_{1}^{-}
$$

Along HJ

$$
j_{1}^{+}=f(L) h_{2}{ }^{+},{h_{2}}^{-}=f(L) j_{1}^{-}
$$

Along JK

$$
k_{1}{ }^{+}=f(L) j_{2}{ }^{+}, j_{2}^{-}=f(L) k_{1}^{-}
$$

Along KN

$$
\boldsymbol{n}^{+}=f\left(L_{2}\right) \boldsymbol{k}_{2}{ }^{+}, \boldsymbol{k}_{2}^{-}=f\left(L_{2}\right) \boldsymbol{n}^{-}
$$

Assuming the generated waves caused by the external transverse force and bending moment are $g_{11}$ and $g_{12}$. This yields propagation relationships along beam $\mathrm{AB}$ as,

Along AG

$$
g_{11}{ }^{+}=f\left(L_{11}\right) a^{+}, \quad a^{-}=f\left(L_{11}\right) g_{11}{ }^{-},
$$

Along GB

$$
\boldsymbol{b}_{\mathbf{1}}{ }^{+}=f\left(L_{12}\right) \boldsymbol{g}_{\mathbf{1 2}}{ }^{+}, \quad \boldsymbol{g}_{\mathbf{1 2}}{ }^{-}=f\left(L_{12}\right) \boldsymbol{b}_{\mathbf{1}}^{-}
$$

The relation between the excited wave amplitudes and applied loading is,

$$
\begin{aligned}
& g_{12}{ }^{+}-g_{11}{ }^{+}=q+m \\
& g_{12}{ }^{-}-g_{11}{ }^{-}=-q+m
\end{aligned}
$$


Equations (40)-(61) can be written in matrix algebraic form as,

$$
A_{f} z_{f}=\boldsymbol{F}
$$

where $A_{f}$ is an $80 \times 80$ coefficient matrix, $z_{f}$ is an $80 \times 1$ component vector, and $\boldsymbol{F}$ is an $80 \times 1$ vector holding the external transverse forces and bending moment.

For the frequency response analyses presented later, two types of loading are applied to the host beam, transverse force and bending moment. Assuming the output point is on the beam $\mathrm{KN}$ with a distance $x$ from the point $\mathrm{K}$, the deflection can be obtained from,

$$
y=\left[\begin{array}{ll}
1 & 1
\end{array}\right] f(x) k_{2}{ }^{+}+\left[\begin{array}{ll}
1 & 1
\end{array}\right] f(-x) k_{2}^{-}
$$

Under two types of applied loadings, the deflection can be combined into one expression,

$$
y_{t}=\sqrt{y_{f}^{2}+y_{m}^{2}}
$$

where $y_{f}$ is the deflection of the output point under transverse force loading, and $y_{m}$ is the deflection of the output point under bending moment loading.

Similarly, the deflection of the loading point $\mathrm{G}$ is given by,

$$
x=\left[\begin{array}{ll}
1 & 1
\end{array}\right] g_{11}{ }^{+}+\left[\begin{array}{ll}
1 & 1
\end{array}\right] g_{11}{ }^{-}
$$

Under two types of applied loadings, the deflection of the loading point is,

$$
x_{t}=\sqrt{x_{f}^{2}+x_{m}^{2}}
$$

where $x_{f}$ is the deflection of the loading point under transverse force loading, and $x_{m}$ is the deflection of the loading point under bending moment loading.

Then, the frequency response of the LR beam can be obtained from,

$$
f=20 \lg \left(\frac{y_{t}}{x_{t}}\right)
$$

\section{Numerical and Experimental Results and Discussion}

With the analysis approach illustrated above, several numerical examples were chosen to present the forced vibration response of a finite Timoshenko LR beam carrying eight periodic uncoupled force-moment type resonators. The material of the example host beam is aluminum. The physical properties are as follows: Young's modulus $E=70 \mathrm{GN} / \mathrm{m}^{2}$, Poisson's ratio $v=0.33$, shear modulus $G=E / 2(1+v)$ related to $E$ and the Poisson's ratio $v$, mass density $\rho=2700 \mathrm{~kg} / \mathrm{m}^{3}$, cross-sectional geometry of the host beam given by $3 \times 10^{-3} \times 1 \times 10^{-2} \mathrm{~m}^{2}$, and the shear coefficient is found using $\kappa=10(1+v) /(12+11 v)$. The lattice constant $L=0.1 \mathrm{~m}$, the loading site $L_{11}=0.01 \mathrm{~m}$, $L_{12}=0.09 \mathrm{~m}$, the measured output point is chosen on the host beam with the distance $L_{m}=0.01 \mathrm{~m}$ from the right end, and the distance from the last resonator to the right end of the host beam $L_{2}=L=0.1 \mathrm{~m}$. Note that same two mass-spring systems are attached with the same moment arm for each uncoupled force-moment type resonator. Thus, in this paper, $m_{A}=m_{B}, k_{A}=k_{B}$, and $a=b$.

As is widely known, the band-gaps are determined by the natural frequency of the resonators and lattice constant. For the uncoupled force-moment type resonator, the mass and the length of moment arm of the resonator influences the band-gaps as well. Figure 6 plots the upper and lower boundaries of the band-gap as a function of the mass (Figure 6a) and the length of moment arm (Figure 6b) of a resonator with the same natural frequency $227 \mathrm{~Hz}$. In Figure 6a, the stiffness of the spring $k_{A}=k_{B}=m_{A} \times(227 \times 2 \pi)^{2}$. In Figure $6 \mathrm{~b}$, the mass of the resonator is $m_{A}=m_{B}=4.027 \mathrm{~g}$, and the stiffness $k_{A}=k_{B}=m_{A} \times(227 \times 2 \pi)^{2}=8.1921 \times 10^{3} \mathrm{~N} / \mathrm{m}$. As expected, the center frequencies 
of the band-gaps are $227 \mathrm{~Hz}$, which matches the natural frequency of the resonator. From Figure $6 \mathrm{a}$, it can be observed that the bandwidth of the band-gap increases with the increase in the mass of the resonator $m_{A}$ and $m_{B}$. The bandwidth is about 10.28 times greater with $m_{A}=m_{B}=10 \mathrm{~g}$ than $m_{A}=m_{B}=1 \mathrm{~g}$. When the moment arm of the uncoupled force-moment resonator increases, the width of band-gaps increases as well (see Figure $6 b$ ). The bandwidth is about 2.04 times wider when the moment arm $a=b=0.05 \mathrm{~m}$ than $a=b=0.01 \mathrm{~m}$. It is obvious that it is more efficient to broaden the band-gaps of the LR beam through increasing the mass of the uncoupled force-moment type resonator than the moment arm. In general, for this LR beam with uncoupled force-moment type resonators, one can obtain the wider band-gaps of the LR beam through increasing the mass and the moment arm of the resonator.
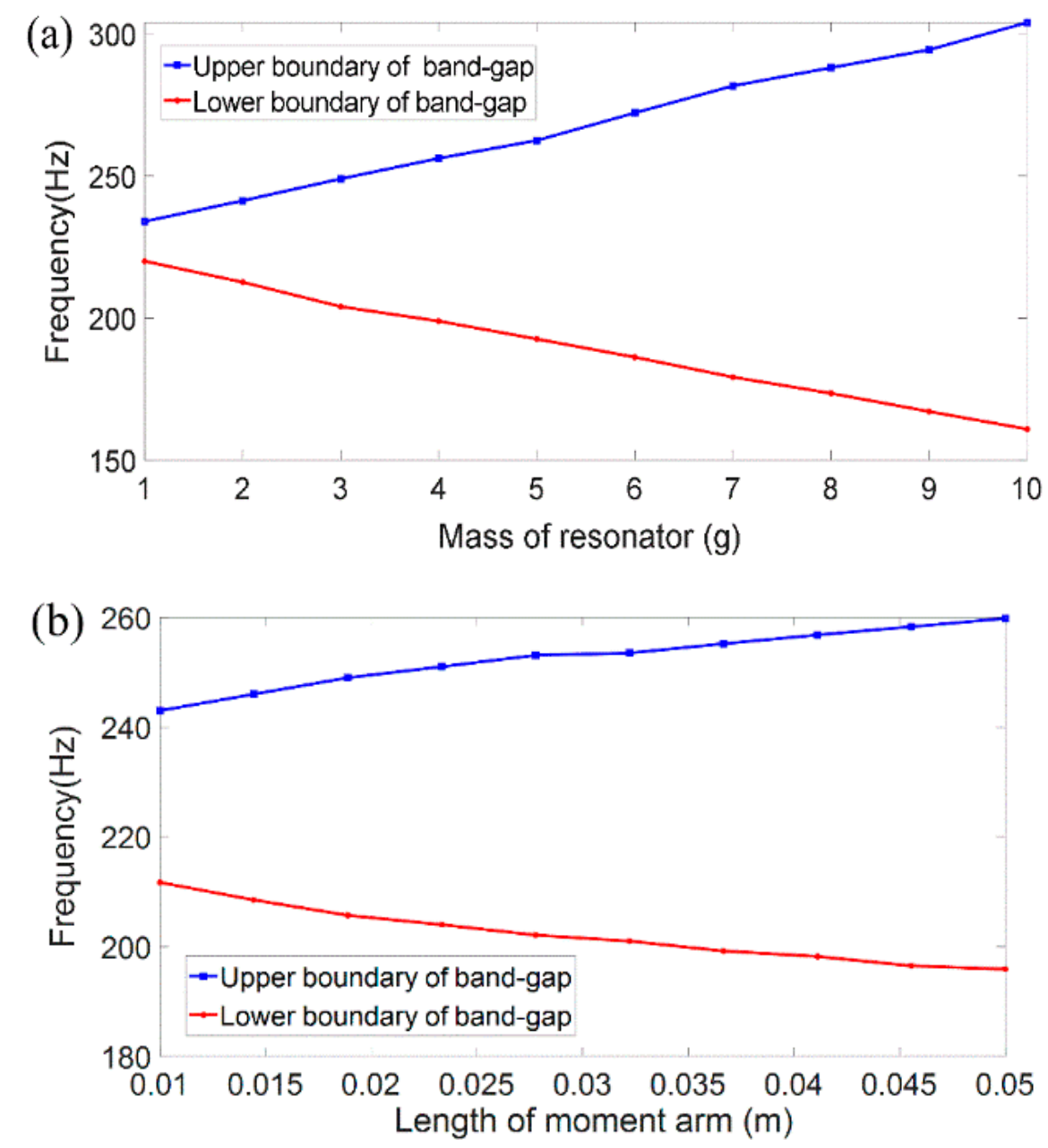

Figure 6. (a) The upper and lower boundaries of the band-gaps as a function of (a) the mass and (b) the moment arm of a resonator with the same natural frequency of the resonator $227 \mathrm{~Hz}$.

A finite Timoshenko LR beam suspended periodically with eight beam-like uncoupled force-moment resonators was modeled and fabricated with the same physical properties as above. A numerical simulation and a vibration experiment were performed to verify the results calculated with the wave-based vibration analysis approach. The material of the beam-like resonator we designed is aluminum, and the model is shown in Figure 7. Similarly to the theoretical analysis, two sets of resonators were chosen with the natural frequency around $227 \mathrm{~Hz}$. The stiffness of the beam-like resonator can be expressed as,

$$
k=\frac{3 E I}{a^{3}}
$$


where $E$ is Young's modulus, $I$ is area moment of inertia, and $a$ is the length of moment arm of the uncoupled force-moment type resonator. The physical parameters of the resonators are summarized in Table 1. In which, $w$ and $h$ are the width and height of the resonator, respectively. $m$ presents the mass of the resonator. $f_{n}$ denotes the natural frequency of the resonator. The beam-like resonators are used because they are much easier to realize in practical engineering, and have the potential for other applications, such as vibration energy harvesting, which is an interesting topic in our future studies.

Table 1. Physical parameters of beam-like resonator samples.

\begin{tabular}{cccccc}
\hline Parameters & $\boldsymbol{a}(\mathbf{m m})$ & $\boldsymbol{w}(\mathbf{m m})$ & $\boldsymbol{h}(\mathbf{m m})$ & $\boldsymbol{m} \mathbf{( g )}$ & $f_{n}(\mathbf{H z})$ \\
\hline Resonator 1 & 25 & 10 & 0.7 & 2.0135 & 225 \\
Resonator 2 & 40 & 10 & 1.44 & 4.027 & 228 \\
\hline
\end{tabular}

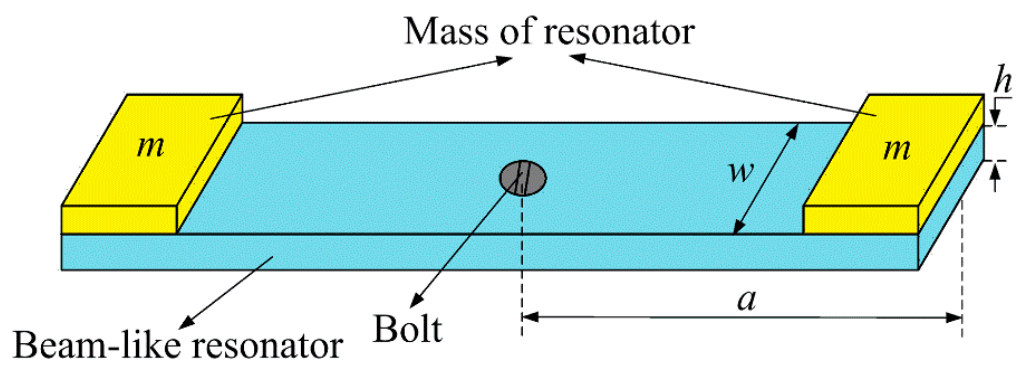

Figure 7. Schematic diagram of the designed beam-like uncoupled force-moment type resonator.

Simulation analysis of the LR beam was carried out based on a FEM (ANSYS Workbench software 2020R2-ANSYS Inc., Canonsburg, PA, USA). In the simulation, the beam was vibrated freely without any boundary limitation at both ends. Harmonic force excitation with a bandwidth that ranges from 0 to $454 \mathrm{~Hz}$ was loaded in the $y$ direction on the host beam with a distance $L_{11}=0.01 \mathrm{~m}$ from the left end. The output acceleration was measured at the point with the distance $L_{m}=0.01 \mathrm{~m}$ from the right end on the host beam. One of the structural models in the simulation is described in Figure 8, in which the red arrow indicates harmonic excitation.

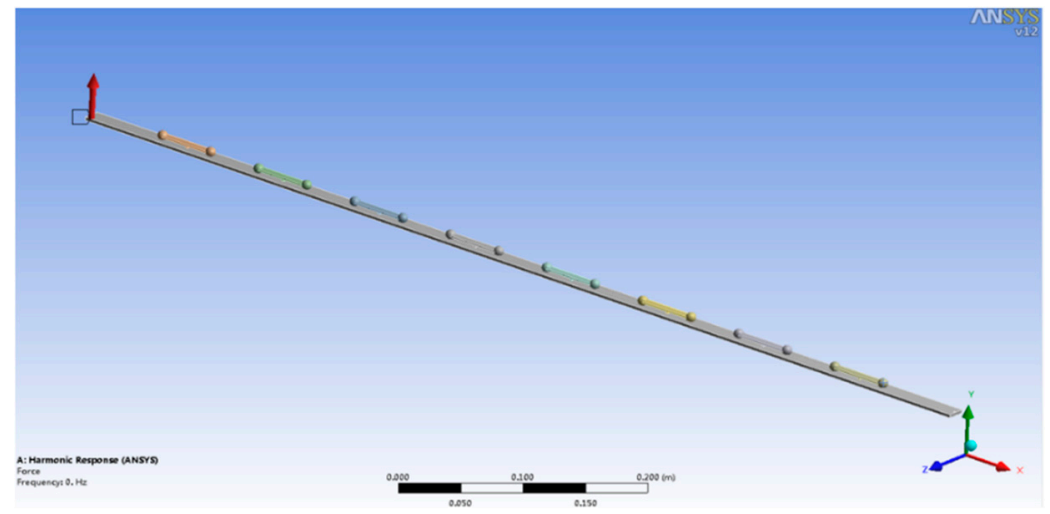

Figure 8. Structural model in the finite element method (FEM) simulation.

The experimental setup is depicted in Figure 9. In the experimental configuration, the host beam was suspended from the ceiling by two elastic straps, which provided a free-free end boundary condition to the beam. The white-noise signal with the amplitude of acceleration $1 \mathrm{~cm} / \mathrm{s}^{2}$ ranged from $0 \mathrm{~Hz}$ to $450 \mathrm{~Hz}$ was generated by the function generator (LMS Test. Lab software 18a-LMS Inc., Leuven, Belgium) and then fed to the vibration shaker through a power amplifier. Excitation was loaded at the same point with the same direction on the host beam as the one in the simulation (as shown in Figure 8). Two vibration accelerometers were attached at the load and measured points 
on the LR beam to measure the input and output accelerations, respectively. One period of a test sample in the experiment is presented in Figure 10. The mass of the resonator was realized by an aluminum block.

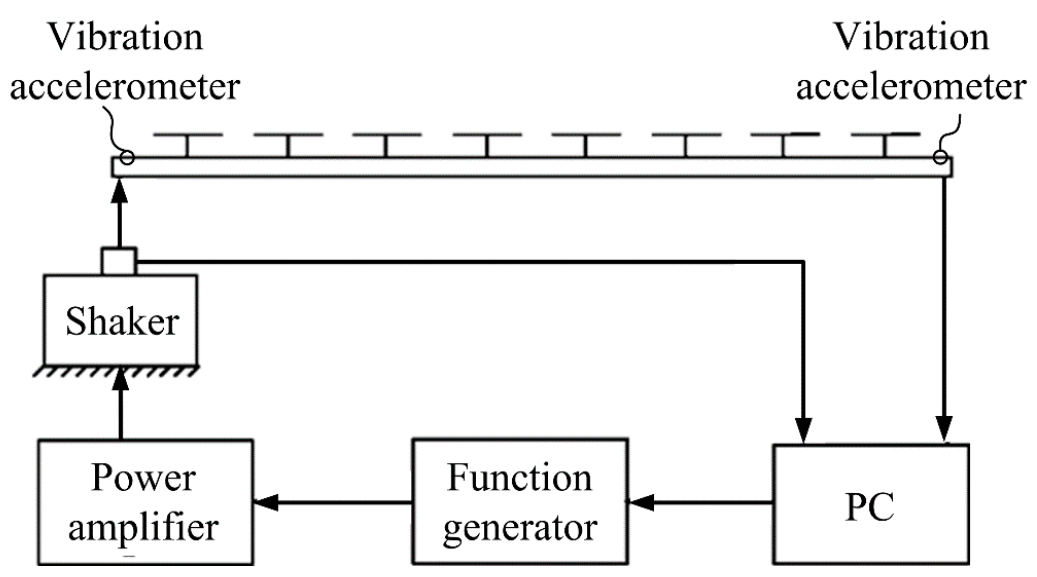

Figure 9. Experimental setup for measuring the input and output accelerations near the two ends of the host beam.

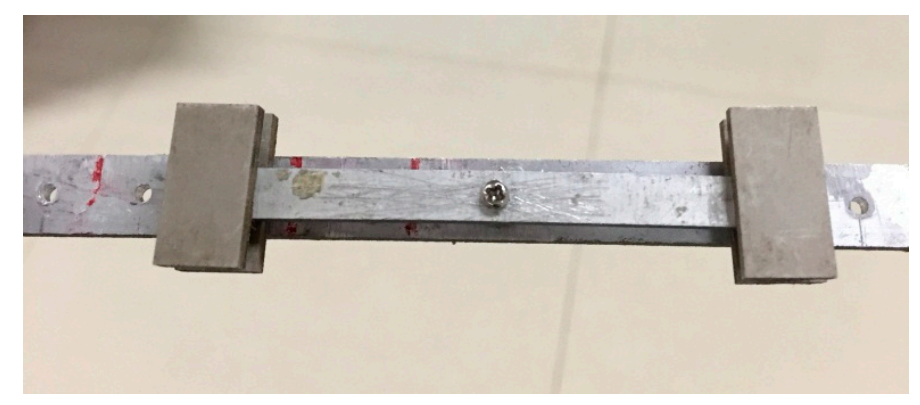

Figure 10. One period of the test specimens.

Figure 11a,b show the numerical and measured frequency respond functions (FRFs) of the LR beam with periodic resonator 1 and resonator 2, respectively. Consider the propagation attenuation of the wave in the experiment, the flexural vibration band-gap in the measured transmission frequency respond curve is defined as the frequency range below $-10 \mathrm{~dB}$, while the one is defined below $0 \mathrm{~dB}$ line for the theoretical curves. Thus, the measured band-gap ranges are $206 \sim 245 \mathrm{~Hz}$ (resonator 1) and 187 $285 \mathrm{~Hz}$ (resonator 2), respectively. The measured and simulated band-gaps show good agreement with the theoretical results obtained through the wave-based vibration analysis approach. Moreover, the influence of mass and length of the moment arm of the resonator on the width of the band-gaps is verified by the measured results. Comparing the curves of Figure 11a,b (solid lines), the width of band-gap is broadened about $151 \%$ when a $100 \%$ increase in the mass and $60 \%$ increase in the length of moment arm of the resonator occurs. The result demonstrates the method to obtain wide band-gaps easily in the low-frequency range for the LR beam suspended with an uncoupled force-moment type resonator in practical applications. 

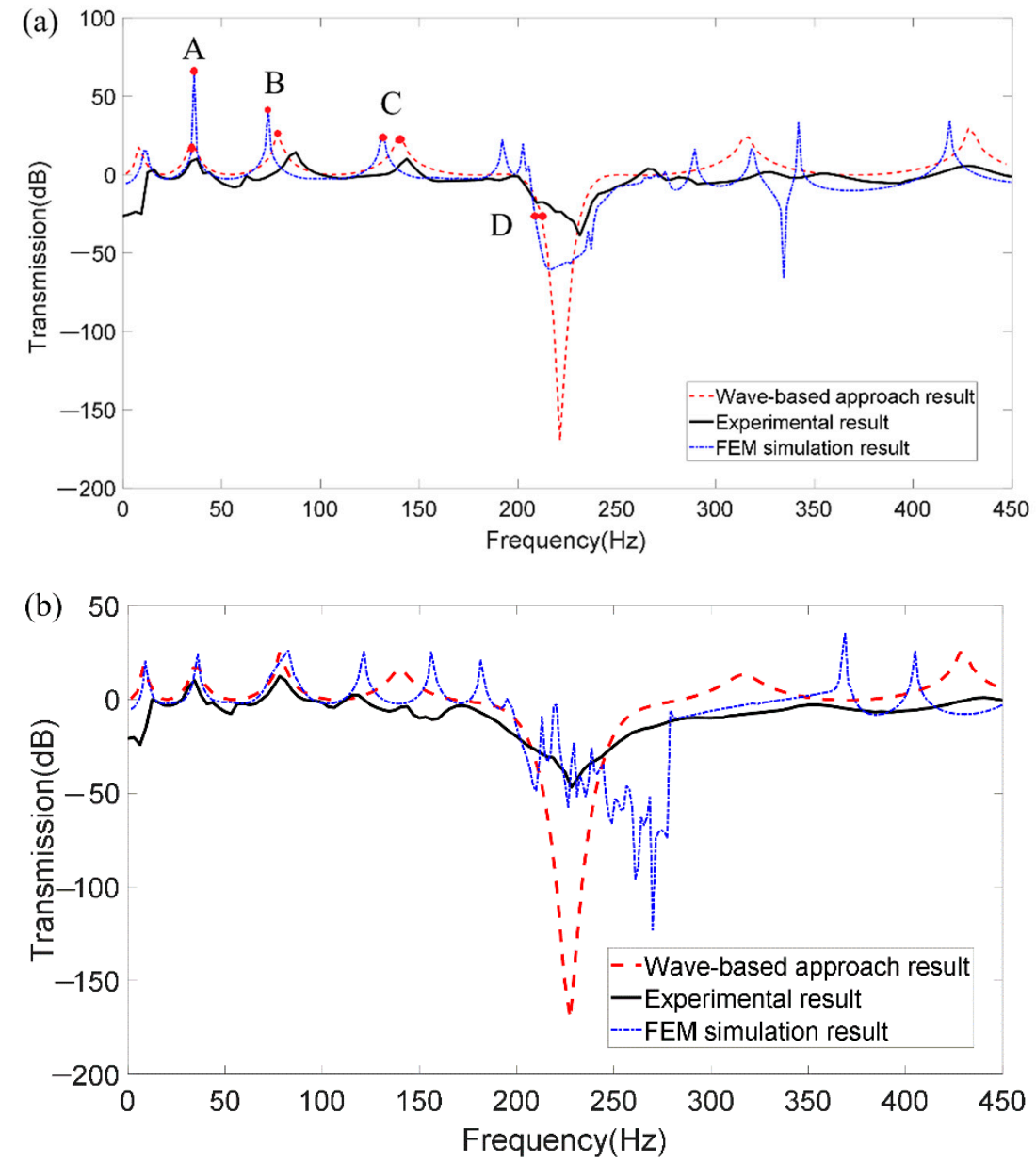

Figure 11. The numerical and measured frequency response curves of (a) resonator 1, and (b) resonator 2.

Note that there are some extra resonance peaks in the measured results (see solid lines in Figure 11a, near 60, 260, 280, and $356 \mathrm{~Hz}$, and in Figure 11b, near 60, 96, 115, and $153 \mathrm{~Hz}$ ). It is because the uncoupled force-moment type resonator in our experiment is realized by a beam-like structure, which provides more vibration mode shapes than a simple spring-mass resonator, such as transverse deflection and rotation. It is also verified by the extra resonance peaks in the simulated curves obtained from FEM (see the dot dash line in Figure 11a,b). Because the band-gaps of the LR beam are the most important properties we were concerned about in practical applications, and all the extra resonance peaks are either near or below the $0 \mathrm{~dB}$ line, the unconformities are negligible.

To better understand the behavior of the structure, four points on the FRF curves in Figure 11 were selected to analyze the steady-state vibration profiles of the LR beam as presented in Figure 12. Two methods were used in Figure 12 as a comparison. The results show good agreement between vibration profiles and frequencies. There are small differences in the frequencies obtained by the two methods. This expected difference, as discussed above, is because the uncoupled force-moment type resonator in the simulation is realized by a beam-like structure, which provides more complex stiffness than a simple spring-mass resonator. Despite the expected difference, the results obtained from wave-based approach and FEM exhibit good agreement. Figure $12 \mathrm{a}-\mathrm{c}$ present the vibration profiles at the resonance peaks A, B, and C. No obvious vibration attenuation along the structure can be observed on the vibration profiles. Figure $12 \mathrm{~d}$ shows the vibration profiles inside the band-gap (around $120 \mathrm{~Hz}$ ), the vibration amplitude at the right end of the LR beam is very small in comparison with that of the left end (excitation point). The vibration is highly localized close to the left end of the structure and is sharply attenuated along the structure, which presents as a band-gap. 
(a)
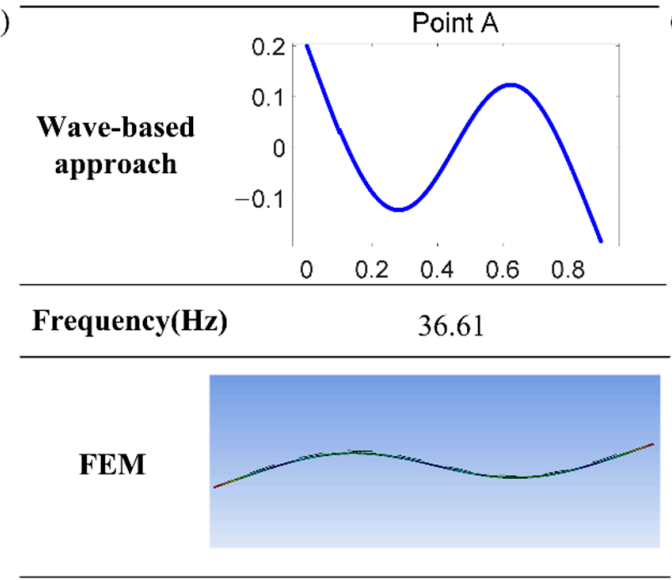

Frequency(Hz)

(c)
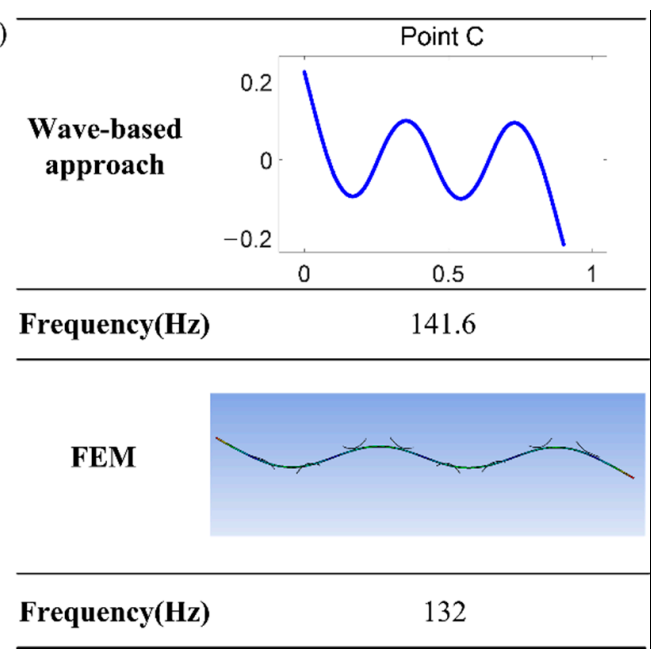

(b)
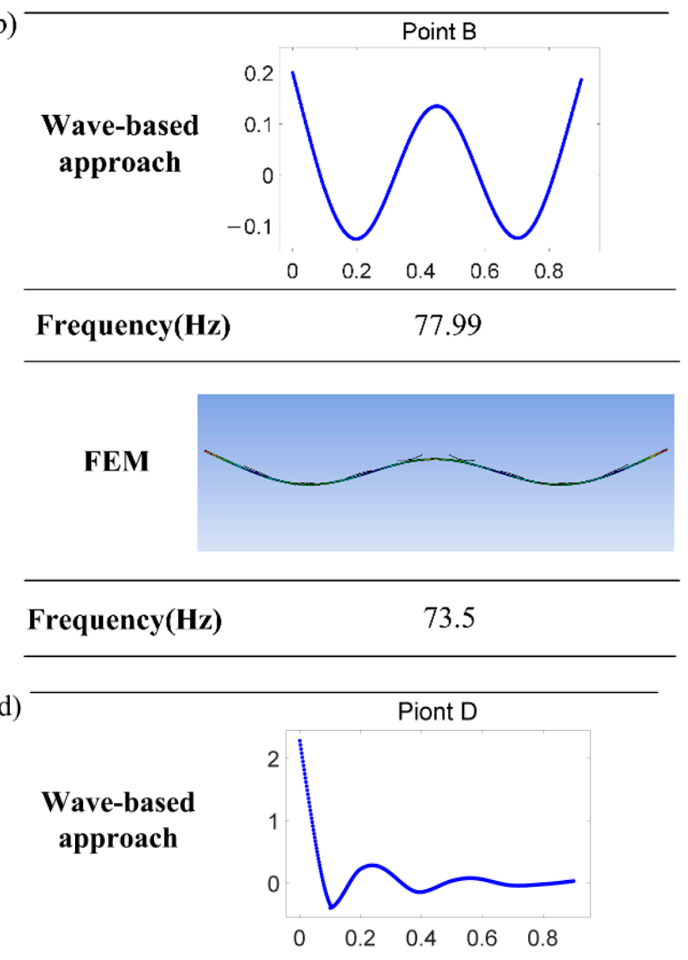

Frequency(Hz) 121.2

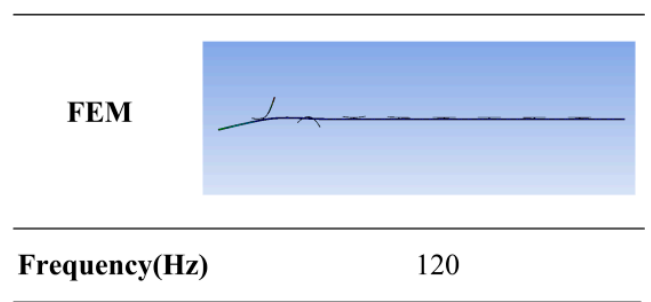

Figure 12. Steady-state vibration profiles obtained using the wave-based analysis approach and the FEM at the frequencies by the points (a) A, (b) B, (c) C, and (d) D in Figure 11a.

\section{Concluding Remarks}

In this paper, a finite Timoshenko LR beam carrying periodic 2-DOF uncoupled force-moment resonators is studied based on a wave-based vibration analysis approach. In formulating the system consisting of a host beam and uncoupled force-moment resonators, each resonator is considered as a source of an external transverse force and a bending moment to the beam. At each point that the resonator was attached, the reflected and transmitted coefficients can be easily assembled as a unit set of equations, which provides an accuracy and efficiency method in design and analysis of a finite Timoshenko LR beam. In addition, the parametric study is investigated to provide a promising method to broaden the low-frequency band-gap, such as adding the mass and length of moment arm of the resonator. Two sets of beam-like resonator samples are fabricated and measured to validate the accuracy of the proposed wave-based analysis method in analyzing frequency response functions of the LR beam. Vibration profiles acquired from wave-based analysis approach and FEM both show the resonance peak and band-gap vibration behavior of the LR beam. Theoretical calculation with the wave-based approach, numerical simulation using FEM, and the experiment all indicate the existence of a low frequency vibration band-gap in the LR beam. Results of this study prove that the wave-base vibration analysis approach is a powerful analytical tool in solving complex vibration problem in periodic LR structures. Future work can apply the proposed method to study the band-gap properties of 2-dimension and 3-dimension LR structures. 
Author Contributions: This paper represents a result of collaborative teamwork. H.L. developed the concept and prepared the manuscript. Y.Z. provided constructive suggestions and revised the manuscript. The two authors contributed equally to this work. All authors have read and agreed to the published version of the manuscript.

Funding: This work was funded by the China Scholarship Council under grant number 201906085003.

Conflicts of Interest: The authors declare no conflict of interest.

\section{References}

1. Mead, D.J. Wave propagation in continuous periodic structures: Research contributions from Southampton. J. Sound Vib. 1996, 190, 495-524. [CrossRef]

2. Sigalas, M.M.; Economou, E. Elastic and acoustic wave band structure. J. Sound Vib. 1992, 158, $377-382$. [CrossRef]

3. Kushwaha, M.S.; Halevi, P.; Dobrzynski, L.; Djafari-Rouhani, B. Acoustic band structure of periodic elastic composites. Phys. Rev. Lett. 1993, 71, 2022-2025. [CrossRef] [PubMed]

4. Cao, D.; Hu, W.; Gao, Y.; Guo, X. Vibration and energy harvesting performance of a piezoelectric phononic crystal beam. Smart Mater. Struct. 2019, 28, 085014. [CrossRef]

5. Qiao, H.; Li, Q.S.; Li, G.Q. Vibratory characteristics of flexural non-uniform Euler-Bernoulli beams carrying an arbitrary number of spring-mass systems. Int. J. Mech. Sci. 2002, 44, 725-743. [CrossRef]

6. Yu, D.; Liu, Y.; Zhao, H.; Wang, G.; Qiu, J. Flexural vibration band gaps in Euler-Bernoulli beams with locally resonant structures with two degrees of freedom. Phys. Rev. B 2006, 73, 064301. [CrossRef]

7. Yu, D.; Liu, Y.; Wang, G.; Zhao, H.; Qiu, J. Flexural vibration band gaps in Timoshenko beams with locally resonant structures. J. Appl. Phys. 2006, 100, 124901. [CrossRef]

8. Wang, M.Y.; Wang, X. Frequency band structure of locally resonant periodic flexural beams suspended with force-moment resonators. J. Phys. D Appl. Phys. 2013, 46, 255502. [CrossRef]

9. Chen, S.; Song, Y.; Zhang, H. Wave Propagation in L-Shape Beams with Piezoelectric Shunting Arrays. Shock Vib. 2019, 2019, 1-14. [CrossRef]

10. Zhou, J.; Dou, L.; Wang, K.; Xu, D.; Ouyang, H. A nonlinear resonator with inertial amplification for very low-frequency flexural wave attenuations in beams. Nonlinear Dyn. 2019, 96, 647-665. [CrossRef]

11. Xiao, Y.; Wen, J.; Wang, G.; Wen, X. Theoretical and experimental study of locally resonant and Bragg band gaps in flexural beams carrying periodic arrays of beam-like resonators. ASME J. Vib. Acoust. 2013, 135, 041006. [CrossRef]

12. Hajhosseini, M.; Rafeeyan, M.; Ebrahimi, S. Vibration band gap analysis of a new periodic beam model using GDQR method. Mech. Res. Commun. 2017, 79, 43-50. [CrossRef]

13. Liang, X.; Wang, T.; Jiang, X.; Liu, Z.; Ruan, Y.; Deng, Y. A Numerical Method for Flexural Vibration Band Gaps in A Phononic Crystal Beam with Locally Resonant Oscillators. Crystals 2019, 9, 293. [CrossRef]

14. Lepidi, M.; Bacigalupo, A. Wave propagation properties of one-dimensional acoustic metamaterials with nonlinear diatomic microstructure. Nonlinear Dyn. 2019, 98, 2711-2735. [CrossRef]

15. Wu, X.; Li, Y.; Zuo, S. The study of a locally resonant beam with aperiodic mass distribution. Appl. Acoust. 2020, 165, 107306. [CrossRef]

16. Mei, C. A Wave-Based Analytical Solution to Free Vibrations in a Combined Euler-Bernoulli Beam/Frame and a Two-Degree-of-Freedom Spring-Mass System. J. Vib. Acoust. 2018, 140, 061001. [CrossRef]

17. Mei, C. Wave Analysis of In-Plane Vibrations of H- and T-shaped Planar Frame Structures. J. Vib. Acoust. 2008, 130, 061004. [CrossRef]

18. Mei, C. In-plane Vibrations of Classical Planar Frame Structures-An Exact Wave-based Analytical Solution. J. Vib. Control. 2010, 16, 1265-1285. [CrossRef]

19. Mei, C. Wave Analysis of In-Plane Vibrations of L-Shaped and Portal Planar Frame Structures. J. Vib. Acoust. 2012, 134, 021011. [CrossRef]

20. Mei, C. Free vibration analysis of classical single-story multi-bay planar frames. J. Vib. Control. 2012, 19, 2022-2035. [CrossRef]

21. Mei, C.; Mace, B.R. Wave Reflection and Transmission in Timoshenko Beams and Wave Analysis of Timoshenko Beam Structures. J. Vib. Acoust. 2005, 127, 382-394. [CrossRef]

22. Leamy, M.J. Exact wave-based Bloch analysis procedure for investigating wave propagation in two-dimensional periodic lattices. J. Sound Vib. 2012, 331, 1580-1596. [CrossRef] 
23. Salleh, H.; Brennan, M.J. Control of flexural waves on a beam using a vibration neutraliser: Effects of different attachment configurations. J. Sound Vib. 2007, 303, 501-514. [CrossRef]

24. Graff, K.F. Wave Motion in Elastic Soilds; Ohio State University Press: Columbus, OH, USA, 1975.

Publisher's Note: MDPI stays neutral with regard to jurisdictional claims in published maps and institutional affiliations.

(C) 2020 by the authors. Licensee MDPI, Basel, Switzerland. This article is an open access article distributed under the terms and conditions of the Creative Commons Attribution (CC BY) license (http://creativecommons.org/licenses/by/4.0/). 